\title{
Large-scale homogeneous molecular templates for femtosecond time-resolved studies of the guest-host interaction
}

\author{
P. Siffalovic ${ }^{\mathrm{a}, *}$, M. Michelswirth ${ }^{\mathrm{a}}$, P. Bartz ${ }^{\mathrm{a}}$, B. Decker ${ }^{\mathrm{b}}$, C. Agena ${ }^{\mathrm{b}}$, C. Schäfer ${ }^{\mathrm{b}}$, \\ S. Molter ${ }^{\mathrm{c}}$, R. Ros ${ }^{\mathrm{c}}$, M. Bach ${ }^{\mathrm{d}}$, M. Neumann ${ }^{\mathrm{d}}$, D. Anselmetti ${ }^{\mathrm{c}}$, J. Mattay $^{\mathrm{b}}$, \\ U. Heinzmann ${ }^{\mathrm{a}}$, M. Drescher ${ }^{\mathrm{a}}$ \\ a Molekül und Oberflächenphysik, Fakultät für Physik, Universität Bielefeld, 33615 Bielefeld, Germany \\ ${ }^{\mathrm{b}}$ Organische Chemie I, Fakultät für Chemie, Universität Bielefeld, 33615 Bielefeld, Germany \\ ${ }^{\mathrm{c}}$ Experimentelle Biophysik, Fakultät für Physik, Universität Bielefeld, 33615 Bielefeld, Germany \\ d Elektronenspektroskopie, Fachbereich Physik, Universität Osnabrück, 49069 Osnabrück, Germany
}

Received 15 December 2003; received in revised form 31 March 2004; accepted 1 April 2004

\begin{abstract}
Self-assembled monolayer films based on iodobenzoyloxy-functionalized resorc[4]arenes were prepared on gold substrates to serve as model systems for future time-resolved studies of molecular recognition, a mechanism of outstanding importance in bioorganic systems. The film properties were tested using X-ray photoelectron spectroscopy (XPS), atomic force microscopy (AFM) and imaging ellipsometry. An apparatus for time-resolved electron spectroscopy utilizing femtosecond soft X-ray pulses is capable of detecting iodine core-level photolines and the photoinduced dissociation after ultraviolet illumination. The developed technique holds promise for tracking the temporal evolution of chemical shifts of atomic markers as local probes for the dynamics of the guest-host interaction.
\end{abstract}

Keywords: Molecular recognition; Guest-host interaction; Molecular dynamics; Self-assembled monolayer

\section{Introduction}

Molecular recognition is a profound principle of nature providing selectivity in a complex network of chemical reactions. The central mechanism of molecular recognition is a selective interaction between a guest and a host molecule, where the specificity origi-

\footnotetext{
* Corresponding author. Tel.: +49-521-106-5464; fax: +49-521-106-6001.

E-mail address: siffalov@ physik.uni-bielefeld.de (P. Siffalovic).
}

nates from a combination of chemical and geometrical compatibility between the molecules. The formation of the guest-host complex cannot be regarded as instantaneous, but will follow a temporal evolution, including external and/or internal rearrangement of the geometrical (rotation, isomerization), or electronic (electron transfer) structure of one or both constituents. An observation of the dynamics of such intermolecular processes will reveal detailed information on this biologically as well as biotechnologically relevant mechanism. 
According to the different processes involved, molecular recognition generally evolves on a wide scale of times. Molecular dynamics on a millisecond to picosecond time-scale can be temporally resolved using techniques like Förster-resonance-transfer (FRET) (Jares-Erijman and Jovin, 2003; Selvin, 2000), or nuclear magnetic resonance (NMR) and electron paramagnetic resonance (EPR) (Clancy et al., 1998; Myers and Oas, 2002). The most fundamental reactions of this molecular interplay, however, occur in extremely short times down to the femtosecond ( $1 \mathrm{fs}=10^{-15} \mathrm{~s}$ ) regime and their observation requires a detection technique of corresponding speediness. One of the complications for time-resolved studies arises from the statistical nature of molecular recognition: in a living system, one usually cannot predict when a bonding will take place. On the other hand, all current techniques capable of resolving dynamics on a femtosecond time-scale rely on a controlled reaction in response to an external trigger. Moreover, the specificity of the studied process should be reflected in local information about the bonding environment. Traditional optical probes, however, are not very specific on an atomic length scale.
In order to gain some degree of control over the guest-host interaction, we consider the use of resorc[4]arenes (Friggeri et al., 1998), a special type of calixarenes, as model systems. The flexible resorc[4]arene cage-type structure (Fig. 1) and the variability of chemical functionalization at the upper rim make these molecules versatile model hosts for in vitro studies of molecular recognition (Faull and Gupta, 2001). For a properly conditioned molecular system consisting of a (model) guest embedded in a resorcarene host, an external light pulse may induce a very rapid change of the bonding environment experienced by the guest molecule, which in turn may respond by a rearrangement of its structure or position, or may even leave the complex. This response to an external trigger is now accessible to a temporal tracking using the pump-probe concept, where a pump-light pulse initiates a process whose evolution is then followed using a delayed probe pulse by subsequently varying the temporal delay. The pump-probe technique has proven to be the essential tool for femtochemistry (Zewail, 1994), where the dynamics of photoreactions is followed in the time domain. However, in contrast to the usual visible- or UV-probe utilized in tradi-

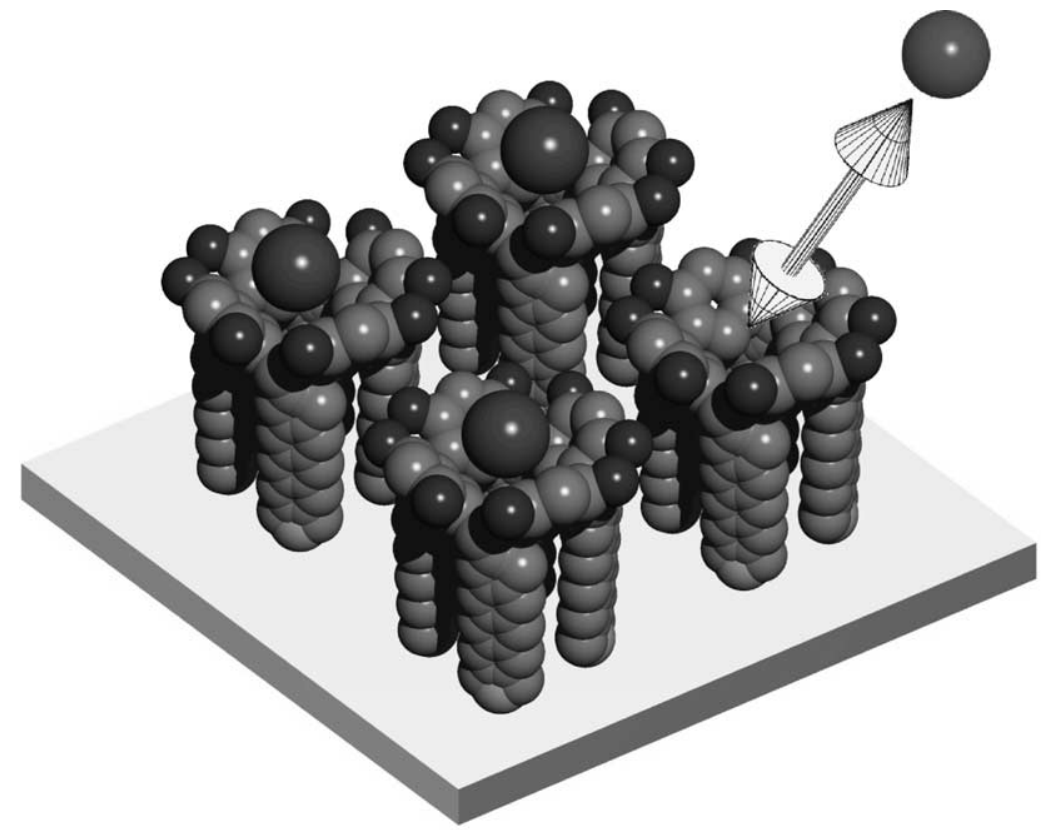

Fig. 1. Schematic drawing of resorc[4]arenes. Their cage-type structure together with a chemically variable upper rim make them versatile model hosts. Self-assembly on a surface provides a dense array of selective bonding sites for guest molecules. 
tional femtochemistry, we intend to use probe pulses in the soft X-ray range thus establishing time-resolved $\mathrm{X}$-ray photoelectron spectroscopy (XPS) (Haight and Peale, 1994; Siffalovic et al., 2001; Drescher et al., 2002a) for studies of intermolecular dynamics. XPS, also termed as electron spectroscopy for chemical analysis (ESCA), is a well-established technique for determining (yet hitherto in a static manner) the elemental composition of a molecule as well as the chemical environment of its atomic constituents (Siegbahn, 1967). Similar to NMR methods, the latter capability relies on the detection of chemical shifts, i.e. spectral shifts reflecting the local electron density around a specific atom within the molecule. The radio- to microwave frequencies of NMR and EPR, however, ultimately determine the temporal resolution of those methods. ESCA instead relies on X-ray frequencies and correspondingly provides femtosecond (Drescher et al., 2001; Siffalovic et al., 2001) and even sub-femtosecond (Hentschel et al., 2001) temporal resolution.

The experimental preconditions and constraints for time-resolved ESCA in the spirit of the scientific goal outlined above are manifold, the most important ones are:

(i) Need of a source of femtosecond soft X-ray (probe) pulses well synchronized to equally short visible or ultraviolet (pump) light pulses. Such a light source on the basis of high harmonic generation (Wahlström et al., 1993) has been realized in our laboratory (Siffalovic et al., 2001) and proven its capability of femtosecond XPS (Siffalovic et al., 2002).

(ii) In ESCA, the information about the process is carried by electrons, which travel only very short distances in solids or liquids before being scattered. Correspondingly, the experiments must be performed at the interface of a molecule with vacuum, which the electrons can escape to.

(iii) Since X-ray pulses from high harmonic generation generally suffer from small intensities (ca. $10^{6}$ photons per second in our case), a high molecular density must be provided. This can be accomplished by preparing the molecular complexes on a solid surface (Davis and Stirling, 1996). (iv) The considered photoinduced processes are nonreversible, therefore each molecule can only be pumped and probed once. On the other hand, a sufficient signal-to-noise ratio will require accumulation over many - typically a few tens of thousands - laser shots. After each individual shot, a pristine spot on the target substrate has to be provided. For the realization of our concept, we therefore have to focus both light pulses down to a small spot of a few tens of $\mu \mathrm{m}$ in diameter and have to cover large (a few $\mathrm{cm}^{2}$ ) substrates of minimal microroughness homogeneously with the molecules under study. By raster-scanning, the substrate with the $50 \mathrm{~Hz}$ repetition rate of the laser system a continuous data acquisition over more than $1 \mathrm{~h}$ can then be sustained.

This article is concerned with the production of such large-scale substrates covered with functionalized resorcarene hosts. We will first describe the synthesis of resorcarenes functionalized with iodine marker atoms and their chemical characterization. The second step involves the preparation of the resorc[4]arenes on a gold surface as a self-assembled monolayer (SAM) film and the film characterization with respect to chemical composition, thickness, density and homogenity. Finally, we will test the utility of the molecular films for ESCA by performing photoelectron spectroscopy on non-illuminated and UV-illuminated substrates. Irradiation with ultraviolet radiation tuned to break the iodine bonds should result in a detectable modification of the surface chemistry.

\section{Methods}

\subsection{Resorcarene synthesis}

The synthetic strategy is shown in Fig. 2. It starts with the acid-catalyzed condensation of resorcinol and 10-undecylenic aldehyde, forming the cyclic tetramer 1a in the rccc configuration. Subsequently, 1a was converted into the tetrasulfide $\mathbf{1 b}$ with 1-decanethiol and 9-BBN (Thoden van Velzen et al., 1995a). In the next synthesis step, octaiodide 1c was prepared. To a suspension of $125 \mathrm{mg}(71.9 \mu \mathrm{mol}) \mathrm{rccc}-2,8,14,20-$ tetra-[n-decyl(n-decylsulphide)]-resorc[4]arene $\mathbf{1 b}$ in 


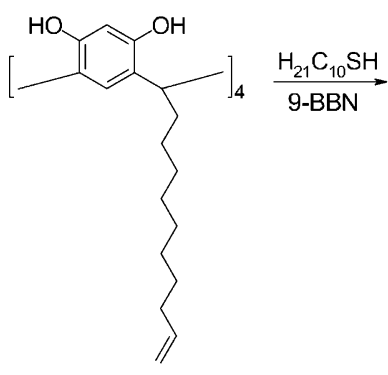

(a)

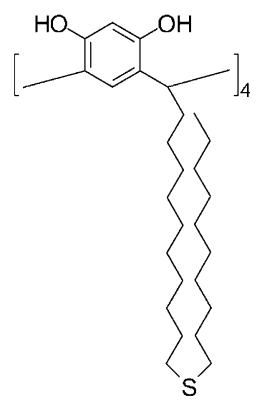

(b)
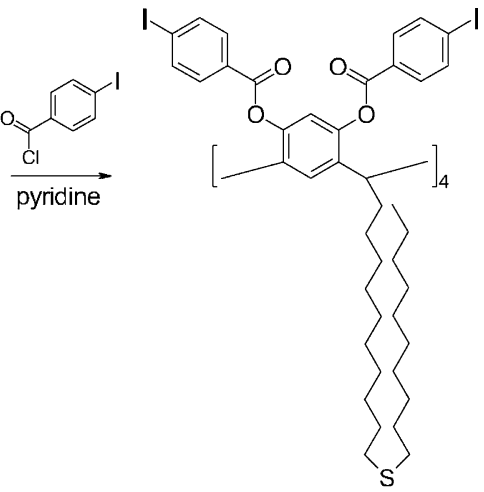

(c)

Fig. 2. Synthesis procedure for resorc[4]arenes with iodobenzyl functionalization and dialkyl sulfide spacers for attachment to a surface.

$900 \mathrm{mg}$ (3.5 mmol) $p$-iodide-benzoic-acid-chloride, $3 \mathrm{~mL}$ pyridine was added. This mixture was heated at $80^{\circ} \mathrm{C}$ for $2 \mathrm{~h}$. The solution was cooled, carefully quenched with water and three times washed with dichloromethane. The combined organic layers were washed with diluted $\mathrm{HCl}$ and water. After drying over $\mathrm{MgSO}_{4}$, the solvent was removed under reduced pressure. The product $(156 \mathrm{mg}, 43.6 \mathrm{mmol}, 61 \%)$ was isolated by HPLC ( $\mathrm{Si}-100$, cyclohexane/ethyl acetate, 97:3) as a colourless solid.

\subsection{Self-assembled monolayer preparation}

Silicon substrates cut from a polished wafer were coated using DC-plasma sputtering. A $40 \mathrm{~nm}$ thick titanium layer was deposited at a rate of $0.24 \mathrm{~nm} / \mathrm{s}$ for improved sticking of the subsequently deposited gold film. Best film qualities were achieved by decreasing the gold deposition rate (Elbel et al., 1995; Wang et al., 1993) in three steps $(128 \mathrm{~nm}$ at $4.4 \mathrm{~nm} / \mathrm{s}$; $75 \mathrm{~nm}$ at $1.25 \mathrm{~nm} / \mathrm{s} ; 70 \mathrm{~nm}$ at $0.37 \mathrm{~nm} / \mathrm{s}$ ). During the whole coating process, the substrate temperature was held at $150^{\circ} \mathrm{C}$. AFM-scans revealed an rmssurface roughness of about $0.9 \mathrm{~nm}$. Self-assembled monolayers of the functionalized resorc[4]arenes on gold were prepared by immersing the substrates directly after evaporation into a $1 \mathrm{mM}$ solution of 1c in ethanol/chloroform (7/3). Self-assembly was achieved by keeping the adsorbate solution containing the gold substrate for $16 \mathrm{~h}$ at $60^{\circ} \mathrm{C}$ and subsequent slow (3h) cooling down to room temperature followed by several rinsings with ethyl alcohol and argon.

\subsection{XPS, AFM and ellipsometry}

X-ray photoelectron spectrocopy (PHI model 5600ci) was performed using the $\mathrm{Al}_{\mathrm{K}}$-line at $1487 \mathrm{eV}$ photon energy to excite inner shells of SAM molecular templates under high-vacuum $\left(10^{-8} \mathrm{mbar}\right)$ conditions. An atomic force microscopy (AFM) capable of covering scan areas of $50 \mu \mathrm{m} \times 50 \mu \mathrm{m}$ (Nanoscope) was used in air to determine the height of assembled resorc[4]arene films. In order to obtain steps between areas with and without SAM, the covered substrates were illuminated with ultraviolet radiation $(\lambda=172 \mathrm{~nm})$ from an excimer-lamp (Ushio) through an etched nickel mesh with $25 \mu \mathrm{m}$ period. Attack from the UV radiation removed the molecular film within $15 \mathrm{~min}$ in the uncovered square areas (Brechling et al., 2004). The UV-microstructured templates were also studied with an imaging nulling ellipsometer (Nanofilm) with $3 \mu \mathrm{m}$ lateral and $0.1 \mathrm{~nm}$ vertical resolution at a wavelength of $658 \mathrm{~nm}$ and an angle of incidence of $60^{\circ}$.

\subsection{Pulsed soft X-ray source and ESCA apparatus}

A home-built titanium-sapphire laser system provides intense ( $200 \mathrm{GW}$ peak power) light pulses of $50 \mathrm{fs}$ duration at a wavelength of $800 \mathrm{~nm}$ and a repetition rate of $50 \mathrm{~Hz}$. Focusing into neon gas at in- 


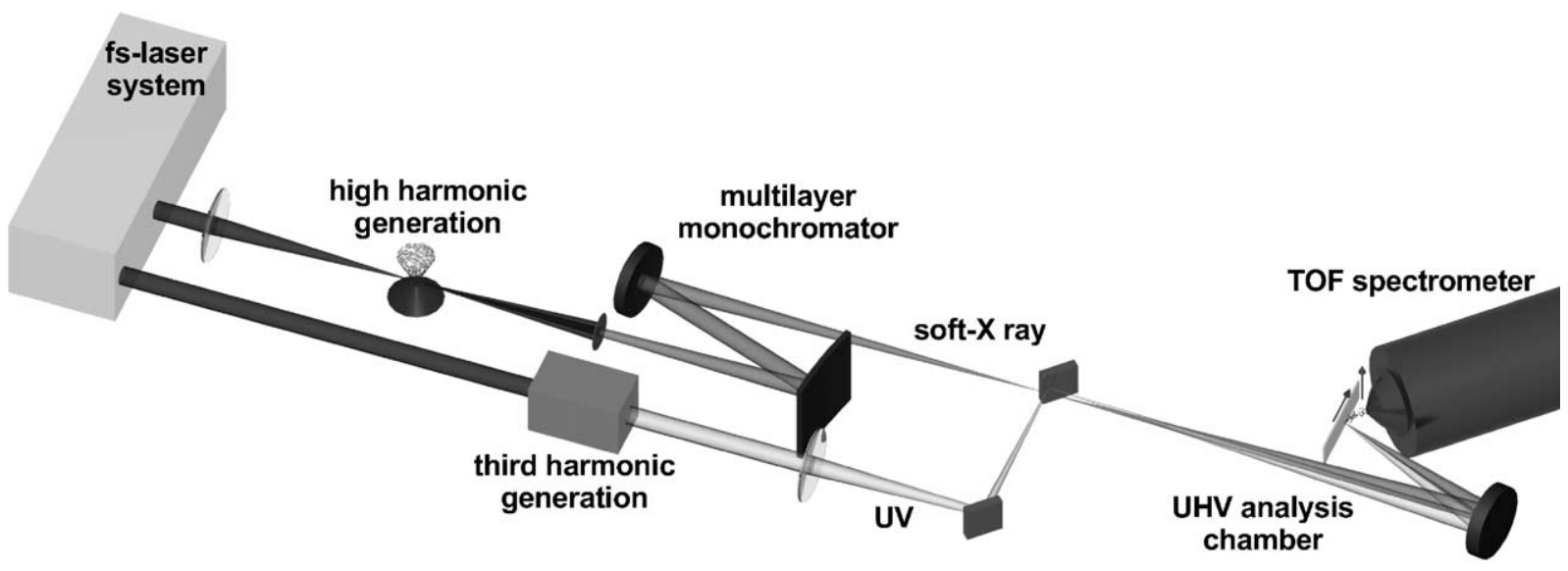

Fig. 3. Apparatus for time-resolved ESCA. Ultrashort soft X-ray pulses are generated as high harmonics of $50 \mathrm{fs}$ laser pulses by focusing into a gas target. After monochromatization the pulses are refocused into a $25 \mu \mathrm{m}$ (FWHM) spot onto a surface together with dissociating UV radiation. The photoemitted electrons are energy-analyzed in a time-of-flight spectrometer.

tensities of $\sim 10^{15} \mathrm{~W} / \mathrm{cm}^{2}$ gives rise to the generation of high harmonic orders of the laser fundamental (Fig. 3). A monochromator based on metal-multilayer Bragg-reflectors (Lim et al., 2001) selects the 45th order corresponding to a photon energy of $70 \mathrm{eV}$ (17.7 $\mathrm{nm}$ wavelength) while preserving the femtosecond time-structure, as has been demonstrated in previous studies on metallic (Siffalovic et al., 2001) or semiconductor surfaces (Siffalovic et al., 2002). This radiation is refocused to a spot of $25 \mu \mathrm{m}$ (FWHM) diameter onto the target under study being located in a ultrahigh-vacuum chamber. Photoemitted electrons from the target are energy-analyzed in a time-offlight (TOF) spectrometer (Drescher et al., 2002b). Frequency tripling of the $800 \mathrm{~nm}$ radiation yields femtosecond ultraviolet pulses at $266 \mathrm{~nm}$, which are spatially overlapped with the soft X-rays.

\section{Results}

\subsection{Characterization of functionalized resorc[4]arenes in solution}

$r c c c$-2,8,14,20-Tetra-[ $n$-decyl-( $n$-decylsulphide)]$4,6,10,12,16,18,22,24$-octa- $O$-( $p$-iodide-benzoyl)resorc[4]arene 1c was characterized by ${ }^{1} \mathrm{H}$ and ${ }^{13} \mathrm{C}$ NMR spectroscopy, ESI-MS spectrometry and UV-vis spectroscopy. In solution the molecule exists in the boat conformation, which could be confirmed by ${ }^{1} \mathrm{H}$ and ${ }^{13} \mathrm{C}$ NMR spectra showing a perfect $\mathrm{C}_{2 v^{-}}$ symmetry. In solution, this conformation is typical for resorcarenes with huge $O$-substituents (Timmermann et al., 1996).

\subsection{Self-assembled monolayer characterization}

XPS provides a quantitative measure of the elemental composition of a surface. The XPS spectrum of the iodine-functionalized resorc[4] arene SAM is shown in Fig. 4. Analysis of the peak areas and consideration of the ionization cross section for the different elements yields a relative abundancy of the elements C:O:I of 1:0.15:0.07. This compares to a ratio of 1:0.10:0.05 from the molecular stoichiometry of the film. Sulfur from the thiolether group constituting the bonding to the surface was also found in the XPS spectrum, but too faint for a quantitative analysis. Prolonged illumination with X-rays at $1487 \mathrm{eV}$ resulted in a deformation of the peak forms, indicating a deterioration of the film due to bond fission or cross-linking (Jäger et al., 1997).

In order to obtain information about the film height from atomic force microscopy, a step between the film surface and the substrate must be provided. To this end, the SAM film was patterned following the procedure described in 2.3. The AFM image (Fig. 5a) was analyzed by selecting 51 separated sub-areas, each 


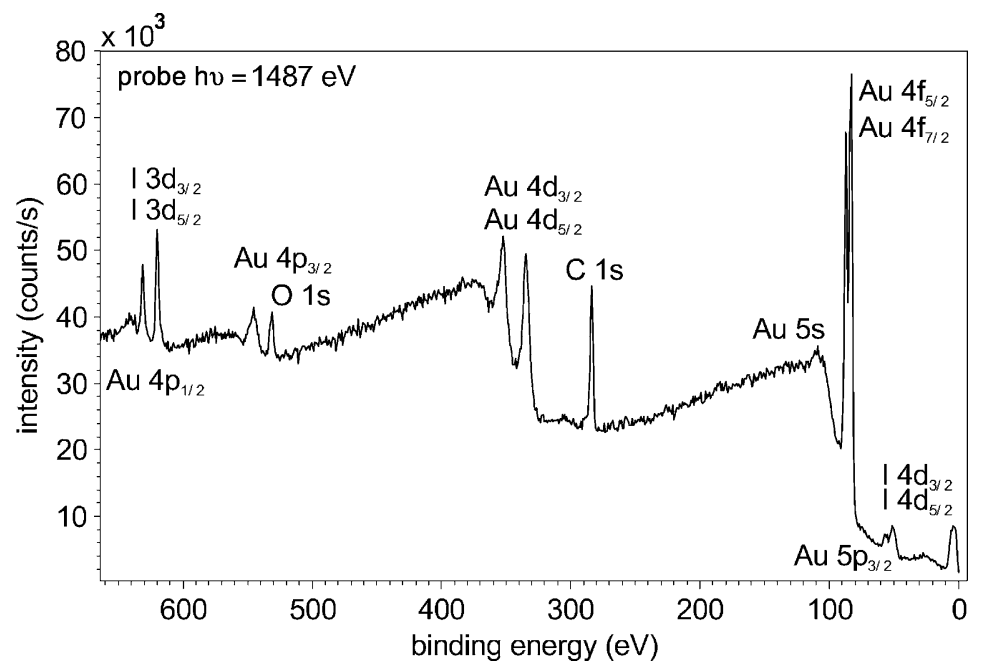

Fig. 4. The stoichiometry of the self-assembled monolayer films of $\mathbf{1 c}$ is extracted from the peak areas of X-ray photoelectron spectra after excitation of the molecular films with $1487 \mathrm{eV}$ radiation.

$0.42 \mu \mathrm{m} \times 21.5 \mu \mathrm{m}$ wide and containing a step between a SAM-covered and an uncovered part. After averaging of line-scans perpendicular to the step a mean step height was determined for each sub-area. The histogramm of these values, shown in Fig. 5b, leads to a height of the SAM film of $2.7 \pm 0.5 \mathrm{~nm}$. Taking into account the height of about $0.4 \mathrm{~nm}$ for the iodobenzyl groups, this is in accordance with literature values (Thoden van Velzen et al., 1995b) from surface plasmon resonance measurements ranging between 2.0 and $2.8 \mathrm{~nm}$ for similar resorc[4]arene tetrasulfides.

Imaging ellipsometry of the UV-patterned films also reveals the lateral structure (Fig. 6), but here the contrast results from a combination of thickness and refractive index. On the basis of the rigid surface binding geometry of a single molecule, we expect a growth model where each molecule forming the SAM has already the height of the closed monolayer. Correspondingly, we can calculate from the measured ellipsometric angles the effective index of refraction, being a function of the film density (Smith, 1968). Combining the thickness information from the AFM measurement with the ellipsometry data yields an effective refractive index of 1.45 . This corresponds to a value previously considered for densely packed calixarene monolayers on gold (Thoden van Velzen et al., 1995b).
In spite of the unique capabilities of X-ray sources based on high harmonic generation in terms of temporal resolution (Hentschel et al., 2001) they generally suffer from low average intensity. Our laser based source provides approximately $10^{6}$ photons per second; this is to be compared with typically $10^{10}$ photons per second for a conventional X-ray tube or even $10^{13}$ photons per second for beamlines at third-generation synchrotron storage rings. In order to test the feasibility of time-resolved ESCA studies on our model host system, we therefore have to demonstrate that the iodine markers of the resorc[4]arene SAM film are visible in the ESCA spectra obtained with this source. An additional challenge is connected with the low photon energy of $70 \mathrm{eV}$ : while providing larger excitation cross-section as compared to hard X-rays, the corresponding low kinetic energies of approximately $15 \mathrm{eV}$ for the $\mathrm{N}$-shell photoelectrons from iodine overlap with an appreciable background from inelastically scattered electrons originating from the organic film and the gold substrate. In spite of the low relative abundance of iodine atoms in the film, the spectrum in Fig. 7 clearly reveals as atomic fingerprints the two fine-structure components of iodine at $50.6 \mathrm{eV}\left(4 \mathrm{~d}_{3 / 2}\right)$ and $48.9 \mathrm{eV}\left(4 \mathrm{~d}_{5 / 2}\right)$ binding energy. No features from the gold substrate are evident in the spectrum, thus supporting a closed molecular film (Alloway et al., 2003). According to gas phase studies (Frietas et al., 

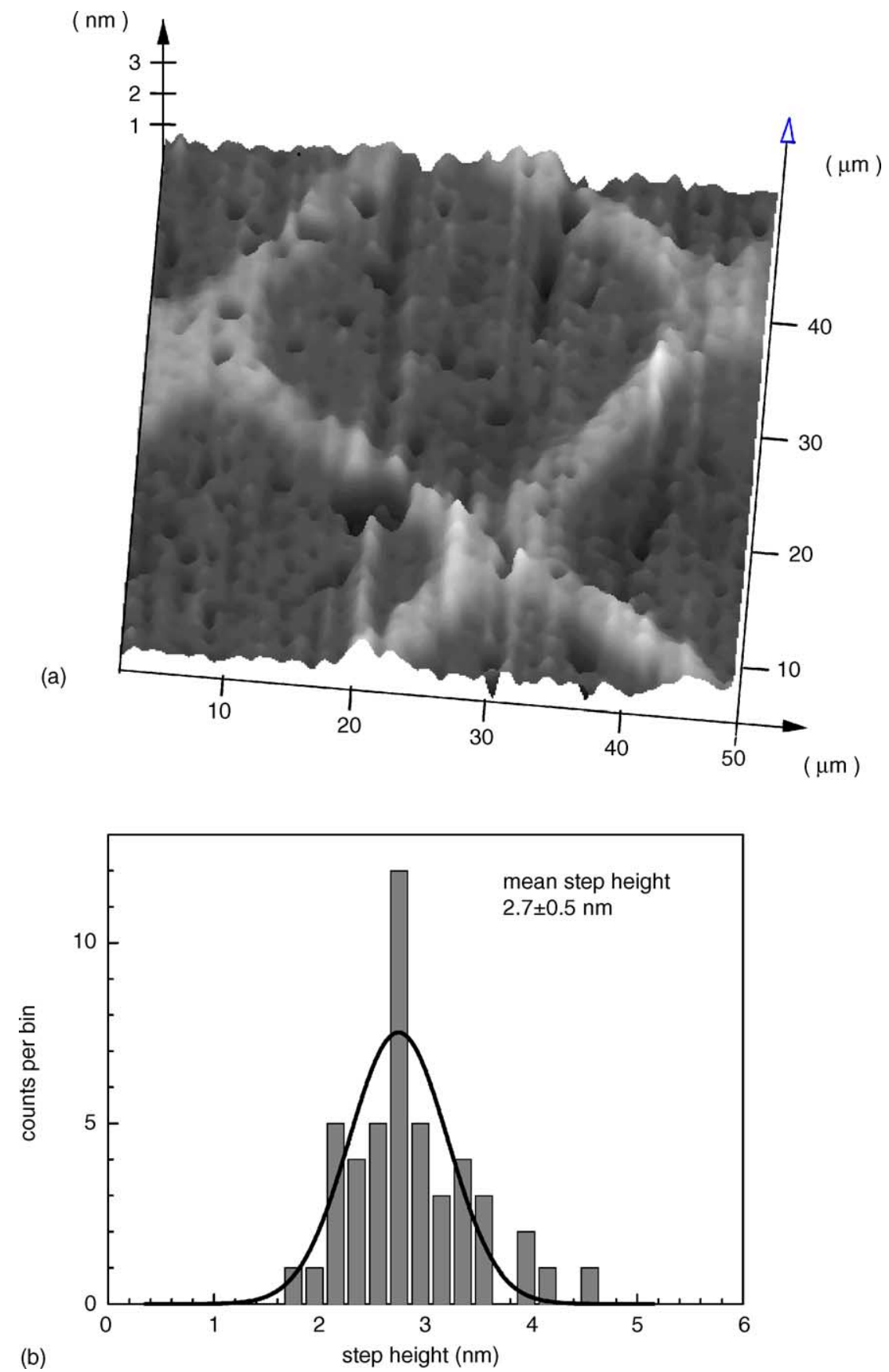

Fig. 5. (a) Atomic force microscopy of a UV-patterned iodine-functionalized resorc[4]arene self-assembled monolayer film yields a height of the molecular film of $2.7 \mathrm{~nm}$. (b) Histogramm of measured step heights in 51 sub-areas of (a). 


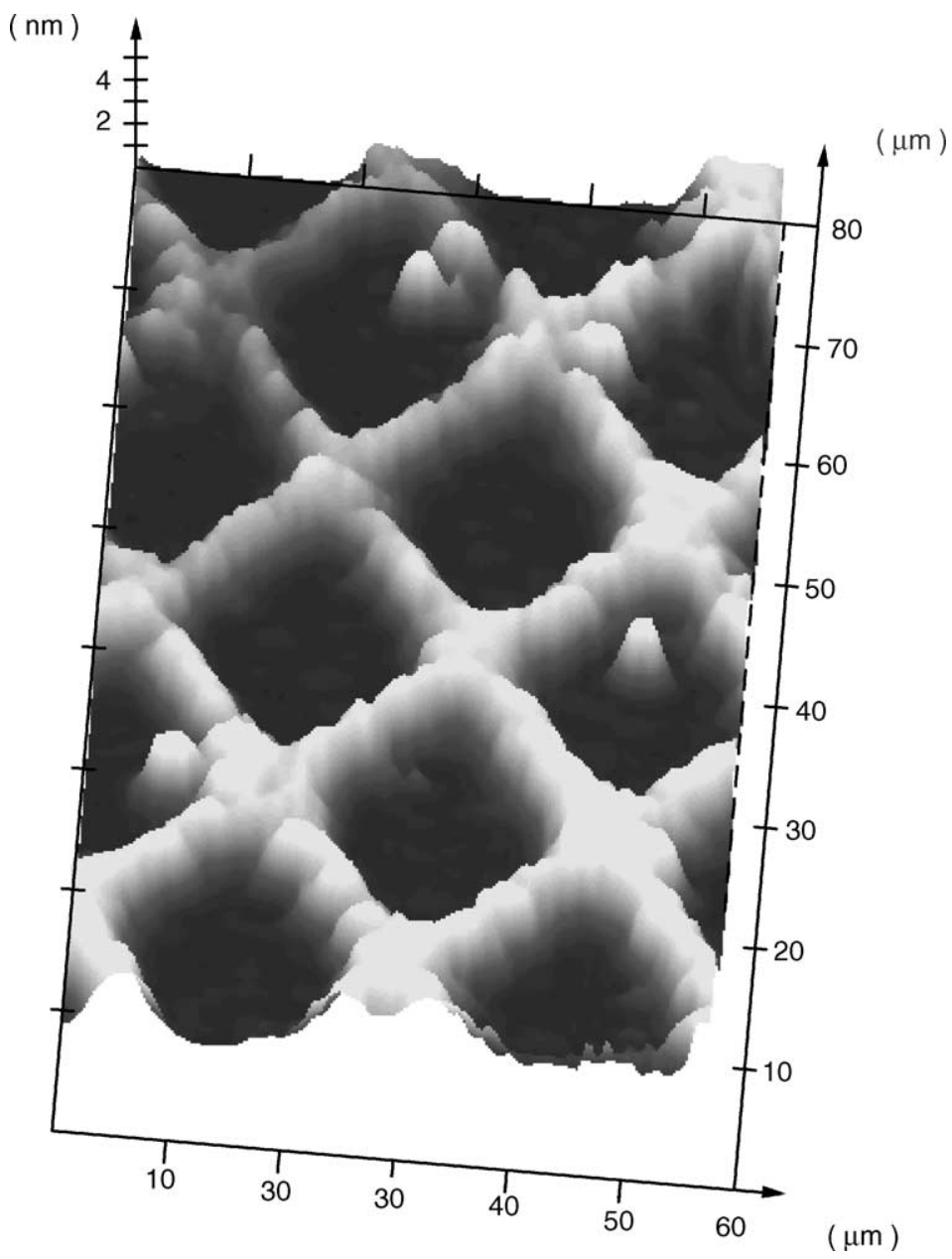

Fig. 6. Imaging ellipsometry of a UV-patterned iodine-functionalized resorc[4]arene self-assembled monolayer film yields an index of refraction of 1.45, if the film height from the AFM study in Fig. 5 is taken into account.

1993; Cheng et al., 1995; Freedman et al., 1980; Kawasaki et al., 1977), illumination of the same spot on the substrate with ultraviolet radiation at $266 \mathrm{~nm}$ wavelength should induce the fission of bonds between the iodine and the benzene group. This behaviour is verified in Fig. 7 also for the iodobenzyl groups being attached to our SAM film. The difference spectrum in Fig. 7 does not show any changes in the valence region thus proving the full integrity of the residual molecular film after iodine photodissociation. The integral under the I $4 \mathrm{~d}$ peaks as a function of the deposited light energy is plotted in Fig. 8. The iodine signal $\mathrm{I}_{S}$ decreases exponentially towards the background $\mathrm{I}_{\mathrm{B}}$ with the characteristic decay constant $\alpha=2.7 \pm 0.4 \mu \mathrm{J}$. $\alpha$ was found independent of the ultraviolet pulse intensity and thus confirms the linear character of the photodissociation even for intensities of the order of $10^{10} \mathrm{~W} / \mathrm{cm}^{2}$. After depositing a dose of $0.28 \mathrm{~J} / \mathrm{cm}^{2}$, the iodine signal almost completely disappears. Assuming a surface density of $7 \times 10^{13}$ molecules $/ \mathrm{cm}^{2}$ corresponding to a densely packed film, this results in a photodissociation cross section of $2.5 \mathrm{Mbarn}$ closely reproducing the previously published $\mathrm{C}_{6} \mathrm{H}_{6} \mathrm{I}$ extinction coefficient (Kavita and Puspendu, 2002). This is a further indication of a closed SAM films with the iodobenzyl groups pointing away from the surface. 


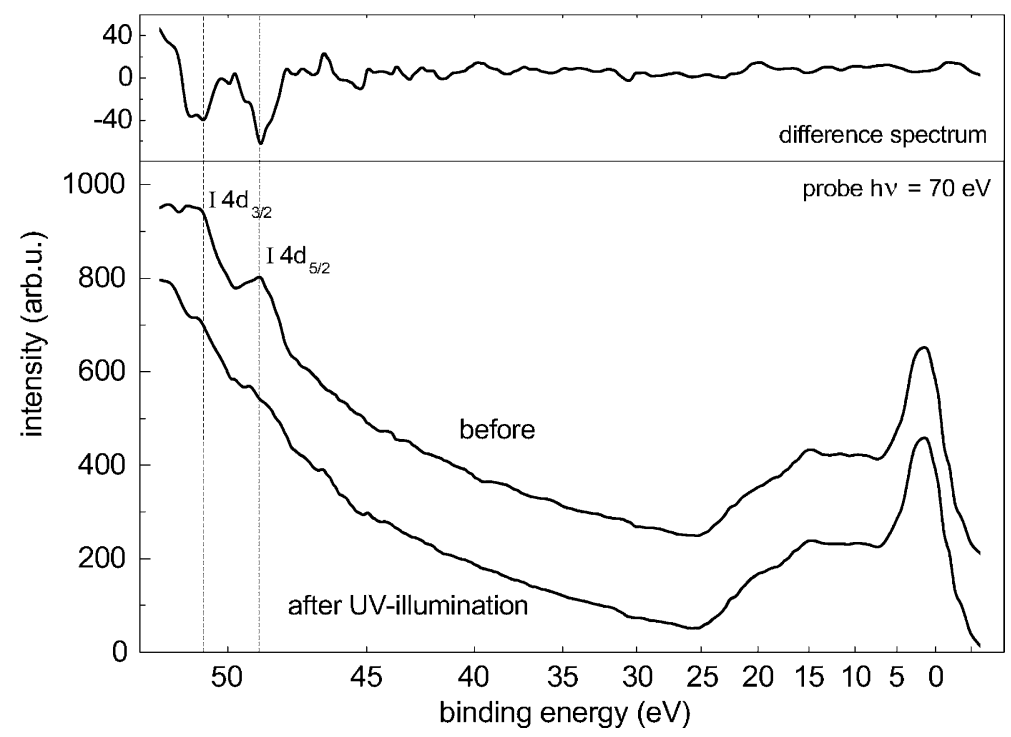

Fig. 7. Photoelectron spectra of a iodine-functionalized resorc[4]arene self-assembled monolayer film obtained with the apparatus from Fig. 3 before (lower panel, top) and after (lower panel, bottom) illumination with ultraviolet radiation at $266 \mathrm{~nm}$. The difference spectrum (upper panel) clearly reveals the disappearance of the iodine atoms from the film while the integrity of the residual molecular layer is preserved.

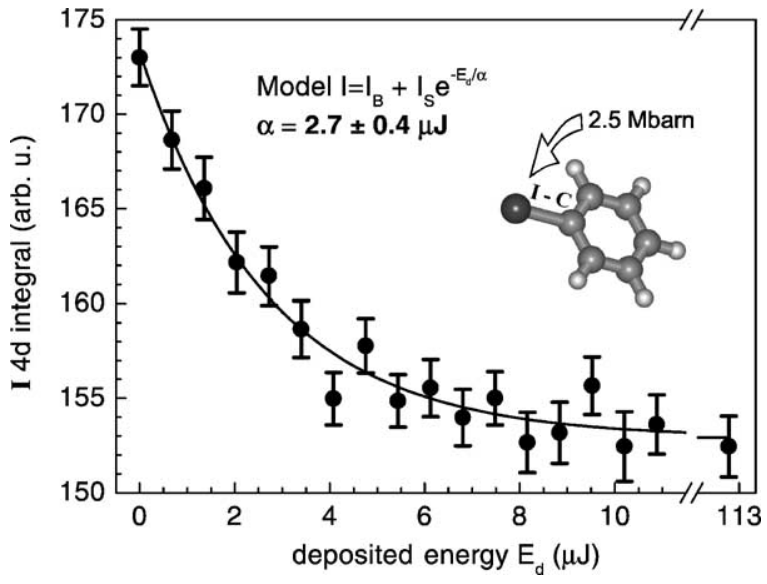

Fig. 8. The iodine signal in ESCA spectra exhibits an exponential dependence on the UV-dose, thus indicating a chemically selective linear photodissociation process.

\section{Discussion}

Resorc[4]arene molecules can be synthesized which carry iodobenzoyloxy functional groups on their upper rim and dialkyl sulfide spacers on their lower rim. Incubation of highly flat gold substrates under suitable conditions gives rise to the formation of self-assembled monolayer films. The stoichiometry is basically preserved upon layer assembly as measured with XPS; an excess oxygen content may be indicative of a water layer on top of the film. AFM studies on UV-patterned films reveal a film thickness close to the expected value for an upright orientation of the molecules and support a proper formation of the film. The films refractive index measured with ellipsometry implies a closed molecular monolayer. ESCA on the prepared molecular templates using a laboratory source for $70 \mathrm{eV}, 50 \mathrm{fs}$ soft-X-ray pulses is feasible and provides sufficient statistical accuracy already after $10 \mathrm{~min}$ of data accumulation. Data acquired on different positions of the substrate do not reveal any changes and thus support the homogeneous preparation over several $\mathrm{cm}^{2}$. Raster scanning of large substrates with laser pump- and X-ray probe pulses focused to microscopic spot size therefore becomes feasible and will enable time-resolved studies of complex molecular systems undergoing non-reversible changes. The spectral signature of the iodine marker atoms attached to the resorc[4]arene is clearly discernible and completely vanishes upon illumination of the film with ultraviolet radiation. The 
spectral signature of selected marker atoms attached to the host (or guest) molecules can now be used to study chemical shifts as a transient probe of the local environment at the guest-host interface when a bond to a guest molecule is formed, breaks apart or transforms in other ways in response to a photoexcitation event.

\section{Acknowledgements}

We are indebted to A. Brechling and U. Kleineberg for supporting us in the UV-patterning and ellipsometry measurements. We gratefully acknowledge the support by the Deutsche Forschungsgemeinschaft.

\section{References}

Alloway, D.M., Hofmann, M., Smith, D.L., Gruhn, N.E., Graham, A.L., Colorado, R., Wysocki, V.H., Lee, T.R., Lee, P.A., Armstrong, N.R., 2003. Interface dipoles arising from selfassembled monolayers on gold: UV-photoemission studies of alkanethiols and partially fluorinated alkanethiols. J. Phys. Chem. B 107, 11690-11699.

Brechling, A., Pohl, M., Kleineberg, U., Heinzmann, U., 2004. Structural organization of DMPC lipid layers on chemically micropatterned seld-assembled monolayers as biomimetic systems. J. Biotechnol. 112, 115-125.

Cheng, P.Y., Zhong, D., Zewail, A.H., 1995. Kinetic-energy, femtosecond resolved reaction dynamics. Modes of dissociation (in iodobenzene) from time-velocity correlations. Chem. Phys. Lett. 237, 399-405.

Clancy, C.M.R., Tarasov, V.F., Forbes, M.D.E., 1998. Timeresolved electron paramagnetic resonance studies in organic photochemistry. Electron. Paramagn. Reson. 16, 50-78.

Davis, F., Stirling, C.J.M., 1996. Calix-4-resorcinarene monolayers and multilayers: formation, structure, and differential adsorption. Langmuir 12, 5365-5374.

Drescher, M., Siffalovic, P., Spieweck, M., Heinzmann, U., 2002a. Applicability of monochromatized high harmonic extended ultraviolet radiation for inner-shell photoelectron spectroscopy. J. Electron. Spectrosc. Relat. Phenom. 127, 103-108.

Drescher, M., Hentschel, M., Kienberger, R., Uiberacker, M., Westerwalbesloh, T., Kleineberg, U., Heinzmann, U., Krausz, F., 2002b. Time-resolved atomic inner-shell spectroscopy. Nature 419, 803-807.

Drescher, M., Hentschel, M., Kienberger, R., Tempea, G., Spielman, C., Reider, G., Corkum, P., Krausz, F., 2001. Xray pulses approaching the Attosecond Frontier. Science 291, 1923-1927.

Elbel, N., Behner, H., von Seggern, H., 1995. Preparation and characterization of epitaxial gold films deposited on mica by direct current magnetron sputtering. J. Vac. Sci. Technol. B 13, 2119-2123.

Faull, J.D., Gupta, V.K., 2001. Selective guest-host association on self-assembled monolayers of calix[4]resorcinarene. Langmuir 17, 1470-1476.

Freedman, A., Yang, S.C., Kawasaki, M., Bersohn, R., 1980. Photodissociation of aryl and aryl-alkyl halides at $193 \mathrm{~nm}$ : fragment translational energy distributions. J. Chem. Phys.72, $1028-1033$.

Frietas, J.E., Hwang, H.J., El-Sayed, M.A., 1993. Molecular rotation clocking of the subpicosecond energy redistribution in molecules falling apart. 2. Excess energy dependence of the rates of energy redistribution in the two photodissociation channels of iodobenzene. J. Phys. Chem. 97, 12481-12484.

Friggeri, A., van Veggel, F.C.J.M., Reinhoudt, D.N., 1998. Selfassembled monolayers of cavitand receptors for the binding of neutral molecules in water. Langmuir 14, 5457-5463.

Haight, R., Peale, D.R., 1994. Tunable photoemission with harmonics of subpicosecond lasers. Rev. Sci. Instrum. 65, 1853-1857.

Hentschel, M., Kienberger, R., Spielmann, C., Reider, G.A., Milosevic, N., Brabec, T., Corkum, P.B., Heinzmann, U., Drescher, M., Krausz, F., 2001. Attosecond metrology. Nature 414, 509-513.

Jäger, B., Schürmann, H., Müller, H.U., Himmel, H.-J., Neumann, M., Grunze, M., Wöll, C., 1997. X-ray and low energy electron induced damage in alkanethiolate monolayers on Au-substrates. Zeitschrift für Physikalische Chemie 202, 263-272.

Jares-Erijman, E.A., Jovin, T.M., 2003. FRET imaging. Nat. Biotechnol. 21, 1387-1395.

Kavita, K., Puspendu, K.D., 2002. Photodissociation of $\mathrm{C}_{6} \mathrm{H}_{6} \mathrm{I}$, $\mathrm{C}_{6} \mathrm{~F}_{5} \mathrm{I}$, and related iodides in the ultraviolet. J. Chem. Phys. 117, 2038-2044.

Kawasaki, M., Lee, S.J., Bersohn, R., 1977. Photodissociation of molecular beams of aryl halides: translational energy distribution of the fragments. J. Chem. Phys. 66, 2647-2655.

Lim, Y.C., Westerwalbesloh, T., Aschentrup, A., Wehmeyer, O., Haindl, G., Kleineberg, U., Heinzmann, U., 2001. Fabrication and characterization of EUV multilayer mirrors optimized for small spectral reflection bandwidth. Appl. Phys. A72, 121-124.

Myers, J.K., Oas, T.G., 2002. Mechanisms of fast protein folding. Annu. Rev. Biochem. 71, 783-815, and references therein.

Selvin, P.R., 2000. The renaissance of fluorescence resonance energy transfer. Nat. Struct. Biol. 7, 730-734.

Siegbahn, K., 1967. ESCA: Atomic, Molecular and Solid State Structure Studied by Means of Electron Spectroscopy. Almqvist \& Wiksells.

Siffalovic, P., Drescher, M., Heinzmann, U., 2002. Femtosecond time-resolved core-level photoelectron spectroscopy tracking surface photovoltage transients on p-GaAs. Europhys. Lett. 60, 924-930.

Siffalovic, P., Drescher, M., Spieweck, M., Wiesenthal, T., Lim, Y.C., Weidner, R., Elizarov, A., Heinzmann, U., 2001. Laserbased apparatus for extended ultraviolet femtosecond timeresolved photoemission spectroscopy. Rev. Sci. Instrum. 72, $30-35$. 
Smith, T., 1968. Ellipsometry for measurements at and below monolayer coverage. J. Opt. Soc. Am. 58, 1069-1079.

Thoden van Velzen, E.U., Engbersen, J.F.J., Reinhoudt, D.N., 1995a. Synthesis of self-assembling resorc[4]arene tetrasulfide adsorbates. Synthesis 8, 989-997.

Thoden van Velzen, E.U., Engbersen, J.F.J., de Lange, P.J., Mahy, J.W.G., Reinhoudt, D.N., 1995b. Self-assembled monolayers of resorcin[4]arene tetrasulfides on gold. J. Am. Chem. Soc. 117, 6853-6862.

Timmermann, P., Verboom, W., Reinhoudt, D.N., 1996. Resorcinarenes. THL 52, 2663-2704.
Wahlström, C.-G., Larsson, J., Persson, A., Starczewski, T., Svanberg, S., Salières, P., Balcou, P., L'Huillier, A., 1993. High-order harmonic generation in rare gases with an intense short-pulse laser. Phys. Rev. A 48, 4709-4720.

Wang, H., Jing, J., Chu, H.T., Henriksen, P.N., 1993. Rearrangement of $\mathrm{Au}\left(\begin{array}{lll}1 & 1 & 1\end{array}\right)$ surface as a result of scanning with scanning tunneling/atomic force microscopes. J. Vac. Sci. Technol. B 11, 2000-2005.

Zewail, A. H., 1994. Femtochemistry - Ultrafast Dynamics of The Chemical Bond, vol. I and II. World Scientific, New Jersey, Singapore. 